Modern Physics Letters A

(C) World Scientific Publishing Company

\title{
Non-singular radiation cosmological models
}

\author{
L. Fernández-Jambrina \\ E.T.S.I. Navales \\ Universidad Politécnica de Madrid \\ Arco de la Victoria $s / n$ \\ E-28040 Madrid, Spain \\ lfernandez@etsin.upm.es \\ http://debin.etsin.upm.es/lfj.htm \\ L.M. González-Romero \\ Departamento de Física Teórica II \\ Facultad de Ciencias Físicas \\ Universidad Complutense de Madrid \\ Avenida Complutense $s / n$ \\ E-28040 Madrid, Spain \\ mgromero@fis.ucm.es
}

\begin{abstract}
In this paper we analyse the possibility of constructing singularity-free inhomogeneous cosmological models with a pure radiation field as matter content. It is shown that the conditions for regularity are very easy to implement and therefore there is a huge number of such spacetimes.
\end{abstract}

Keywords: non-singular; cosmology; geodesic completeness; radiation.

PACS Nos.: 04.20.Dw, 04.20.Ex, 04.20.Jb.

\section{Introduction}

Singularity-free cosmological models were quite popular until the sixties. At that time there were attempts to circumvent initial and final singularities, for instance, by introducing additional fields, such as Hoyle and Narlikar's $C$ field. Such models had the drawback of requiring continuous creation of matter 1]. The interest for that line of research decayed in the seventies when most of the famous singularity theorems were published (cfr. for instance 2,3 ). Under very general and physically realistic conditions these theorems seemed to push cosmological models towards the appearance of singularities, that could be of the Big Bang kind or not. Although most quantum fields, such as Yang-Mills, do not follow them, there was nothing to object to energy conditions. Less criticism would receive causality conditions, which require the absence of closed causal curves. Or the generic condition, which roughly states that the universe is not empty $[3$.

However, in addition to those general and physically acceptable requirements, 
there were other conditions that might be overcome. These were the existence of some causally trapped set in the spacetime, such as closed trapped surfaces 4 or an achronal set without edge 5 . The fulfillment of the latter conditions is not so obvious as for the former ones.

But it was not until the nineties when the first singularity-free cosmological models began to appear $[6$. These models were not realistic, since they were inhomogeneous spacetimes with cylindrical symmetry, but they pointed out that on diminishing the symmetry non-singular behaviour might appear. Since these models were exact solutions in a framework where they are fairly difficult to obtain, it is not strange that the list of such non-singular models was rather short until the end of the century 7 . In fact these spacetimes belonged to larger families among which the absence of singularities required a certain amount of fine tuning 8 . Consequently it was tempting to consider singularity-free cosmological models as a curiosity.

However a large family of regular cosmological models was recently found 9 and did not depend on a finite number of parameters but on two nearly arbitrary functions. This result could mean that although our universe has an initial singularity, it is yet to be explained why alternative, and abundant, possibilities were not considered.

The aim of this paper dates back to Senovilla's original solution. This spacetime had the realistic property of being filled with an incoherent radiation perfect fluid, which is the standard mean matter content for a universe in its early epoch. It is intriguing that when the Senovilla spacetime was extended to include other models with also a linear equation of state 10 , again radiation fluids were the only ones which remained non-singular 10. What we plan to discuss now is the role of radiation in non-singular behaviour. But instead of considering incoherent radiation, we shall focus on pure radiation.

With this aim in mind we devote the next section of this paper to the Einstein equations for radiation fields in inhomogenous cosmological models with a two-dimensional group of isometries acting on spacelike orbits. Section 3 comprises three ways of simplification of the equations. One of them, corresponding to models with a transitivity surface element with lightlike gradient, incorporates additional symmetries to the spacetimes. In Section 4 the analysis of the possible singularities of the families described in the previous sections is completed. A discussion of the results is included at the end of the paper.

\section{Inhomogeneous radiation cosmological models}

We may describe pure radiation by its conserved energy-momentum tensor,

$$
T^{\mu \nu}=\Phi k^{\mu} k^{\nu},
$$

where $k$ is a null vector field describing the lightlike 4-velocity of the radiation, $k^{\mu} k_{\mu}=0$, and $\Phi$ is a positive function related, but not equal, to the radiation energy, 
since this function could be reabsorbed by the vector field $k$ without disturbing the properties of the tensor. Bear in mind that radiation fields are characterised by having all energy-momentum tensor invariants equal to zero.

The energy-momentum conservation equations may be written,

$$
T_{; \nu}^{\mu \nu}=\Phi_{, \nu} k^{\nu} k^{\mu}+\Phi a^{\mu}+\Phi \Theta k^{\mu}=0
$$

in terms of the expansion $\Theta=k_{; \nu}^{\nu}$ and acceleration, $a^{\mu}=k_{; \nu}^{\mu} k^{\nu}$ of the lightlike congruence. Since the acceleration is orthogonal to the radiation velocity, $a$ might have a component parallel to $k$ and a spatial component orthogonal to $k$. The existence of the latter component would mean that the equations would split into components parallel and non-parallel to $k$ and $\Phi$ would be equal to zero. Since this is not our case, $a$ is proportional to $k$ and (2) is a single-component equation.

Now we turn to the geometric description of the spacetime. Considering that except for some spherically symmetric spacetimes 7 every other singularity-free cosmological model has been found within the Ansatz of an Abelian two-dimensional orthogonally transitive group of isometries acting on spacelike orbits, we shall impose that our models share these symmetries. We choose as generators for the symmetries two commuting fields, that we write as $\left\{\partial_{y}, \partial_{z}\right\}$, having in mind the coordinates that we want to use. We take them as mutually orthogonal. There is much freedom for choosing the rest of the coordinates, which we shall name $t, x$. Since every surface admits and isothermal parametrization for which the metric is conformally flat, we take as our $t$ and $x$ the coordinates in such parametrization. With this choice of coordinates, the metric can be written in a suitable form 11,

$$
d s^{2}=e^{2 K}\left(-d t^{2}+d x^{2}\right)+\rho^{2} e^{2 U} d y^{2}+e^{-2 U} d z^{2},
$$

depending on three functions, $K, U$ and $\rho$, of just $t$ and $x$.

As we shall see, there is no possibility for having an axis in these models. Therefore we reject the interpretation of the isometries as cylindrical symmetry and there is no reason a priori for restricting the ranges of the coordinates,

$$
-\infty<t, x, y, z<\infty \text {. }
$$

As an Ansatz we take a velocity of the radiation with no projection on the Killing fields, that is,

$$
k_{ \pm}=e^{-K}\left(\partial_{t} \pm \partial_{x}\right),
$$

where the plus (minus) sign stands for growing (decreasing) coordinate $x$. The normalization factor has been chosen so that $\left\langle k_{+}, k_{-}\right\rangle=-2$.

For such velocities the energy-momentum conservation (2) is written as

$$
\Phi_{t} \pm \Phi_{x}+\Phi\left\{\frac{\rho_{t} \pm \rho_{x}}{\rho}+2\left(K_{t} \pm K_{x}\right)\right\}=0,
$$


which allows a rather simple form in terms of ingoing or outgoing lightlike coordinates,

$$
\left(\partial_{t} \pm \partial_{x}\right)\left\{\Phi e^{2 K} \rho\right\}=0,
$$

for which integration is straightforward,

$$
\Phi e^{2 K} \rho=h(t \mp x),
$$

in terms of a free function $h$ of one outgoing (ingoing) lightlike coordinate.

Finally, the set of Einstein equations for pure radiation can be cast in the form

$$
\begin{aligned}
& U_{t t}-U_{x x}+\frac{1}{\rho}\left(U_{t} \rho_{t}-U_{x} \rho_{x}\right)=0, \\
& \rho_{t t}-\rho_{x x}=0, \\
& K_{t} \rho_{x}+K_{x} \rho_{t}=\rho_{t x}+U_{t} \rho_{x}+U_{x} \rho_{t}+2 \rho U_{t} U_{x} \mp h, \\
& K_{t} \rho_{t}+K_{x} \rho_{x}=\frac{\rho_{t t}+\rho_{x x}}{2}+U_{t} \rho_{t}+U_{x} \rho_{x}+\rho\left(U_{t}^{2}+U_{x}^{2}\right)+h, \\
& K_{x x}-K_{t t}+\frac{U_{x} \rho_{x}-U_{t} \rho_{t}}{\rho}+U_{x}^{2}-U_{t}^{2}=0,
\end{aligned}
$$

where the integrability condition for (9c 9d is the Euler equation (7) and the 1$\mathrm{D}$ inhomogeneous wave equation (9e) for $K$ is a consequence of the rest of the differential system and therefore can be dropped. The remaining set of differential equations for pure radiation models is then reduced to a 2-D wave equation for $U$, a 1-D wave equation for $\rho$ and a quadrature defining $K$.

\section{Simplification of the equations}

The system of differential equations may be simplified by taking $\rho$ as a coordinate for the equations. Three possibilities arise according to the character of the gradient of $\rho$ :

- If the gradient is lightlike, $\rho_{t}=\varepsilon \rho_{x}, \varepsilon^{2}=1$, the 1 -D wave equation is trivially satisfied, $\rho_{x x}=\varepsilon \rho_{t x}=\varepsilon \rho_{x t}=\rho_{t t}$. We have then $\rho=\rho(t+\varepsilon x)$ and the equations defining the quadrature,

$$
\begin{aligned}
& \left(K_{t}+\varepsilon K_{x}\right) \frac{\rho_{t}}{\rho}=\frac{\rho_{t t}}{\rho}+\left(U_{t}+\varepsilon U_{x}\right) \frac{\rho_{t}}{\rho}+2 \varepsilon U_{t} U_{x} \mp \varepsilon \Phi e^{2 K}, \\
& \left(K_{t}+\varepsilon K_{x}\right) \frac{\rho_{t}}{\rho}=\frac{\rho_{t t}}{\rho}+\left(U_{t}+\varepsilon U_{x}\right) \frac{\rho_{t}}{\rho}+U_{t}^{2}+U_{x}^{2}+\Phi e^{2 K},
\end{aligned}
$$

allow us to draw rather constraining consequences,

$$
\begin{aligned}
& \Phi e^{2 K}(1 \pm \varepsilon)+\left(U_{t}-\varepsilon U_{x}\right)^{2}=0, \\
& K_{t} \mp K_{x}=\frac{\rho_{t t}}{\rho_{t}}+2 U_{t}+2 \rho \frac{U_{t}^{2}}{\rho_{t}}+\rho \frac{\Phi e^{2 K}}{\rho_{t}},
\end{aligned}
$$

since $\varepsilon=\mp 1$ in order to have $\Phi \neq 0$ and the gradient of $U$ is also lightlike, $U_{t}=\mp U_{x}$, and parallel to the gradient of $\rho$, but not to the velocity of the radiation. 
No further restrictions are implied for $U$, since it satisfies $U_{t t}=U_{x x}$ and the remaining condition,

$$
0=\rho_{x} U_{x}-\rho_{t} U_{t}=\langle\operatorname{grad} U, \operatorname{grad} \rho\rangle
$$

is trivial.

For $K$ the 1-D wave equation (9e) is no longer inhomogeneous,

$$
K_{t t}-K_{x x}=0,
$$

and we may write the solution in terms of ingoing and outgoing lightlike coordinates, $u=t \mp x, v=t \pm x$,

$$
K(u, v)=f(u)+g(v),
$$

but just $f$ is constrained by the quadrature,

$$
f^{\prime}=\frac{\rho^{\prime \prime}}{2 \rho^{\prime}}+U^{\prime}+\frac{\rho U^{\prime 2}}{\rho^{\prime}}+\frac{h}{2 \rho^{\prime}},
$$

where the prima denotes derivation with respect to $u$.

In principle, we have four independent functions, $f, g, \rho$ and $U$ and the system is solved by prescribing

$$
h=2 \rho^{\prime}\left(f^{\prime}-U^{\prime}\right)-\rho^{\prime \prime}-2 \rho U^{\prime 2} .
$$

The metric can be therefore written as

$$
d s^{2}=-e^{2 f+2 g} d u d v+\rho^{2} e^{2 U} d y^{2}+e^{-2 U} d z^{2},
$$

in terms of lightlike coordinates.

But we see that $f$ and $g$ appear due to just coordinate choices. We may eliminate them by respectively redefining $u$ and $v$ as $\int d u e^{2 f}$ and $\int d v e^{2 g}$ and writing the metric,

$$
d s^{2}=-2 d u d v+F^{2} d y^{2}+G^{2} d z^{2},
$$

in terms of just two independent functions, $F$ and $G$, of $u$.

In this simpler frame the function $\Phi$,

$$
\Phi=-\left(\frac{F^{\prime \prime}}{F}+\frac{G^{\prime \prime}}{G}\right),
$$

just imposes the positiveness requirement.

It is clear that these spacetimes possess more isometries than those which were required from the beginning. Besides the obvious Killing fields, $\left\{\partial_{v}, \partial_{y}, \partial_{z}\right\}$ there are two additional independent isometry generators, $\left\{y \partial_{v}+\int F^{-2} \partial_{y}, z \partial_{v}+\int G^{-2} \partial_{z}\right\}$ and therefore the isometry group is a 
$G_{5}$ acting on null orbits. The spacetimes are not however homogeneous, since the action of the group of isometries is not transitive. The Petrov type is generically $\mathrm{I}$.

The Killing field $\partial_{v}$ is covariantly constant and therefore these models are plane-fronted gravitational waves with parallel rays spacetimes 13, 14, 11. There are examples where the isometry group is even larger, for instance when $F=G$, there is an additional independent Killing field, $y \partial_{z}-z \partial_{y}$. In this case the spacetime is conformally flat.

The results may be extended to non-diagonal metrics,

$$
d s^{2}=-2 d u d v+F^{2} d y^{2}+2 H d y d z+G^{2} d z^{2},
$$

by just introducing another function, $H$, of $u$.

- If the gradient of $\rho$ is spacelike, we may choose $\rho$ as a coordinate, $\rho=x$. Equation (9b) allows us to keep the isothermal parametrization. The system thereby becomes much simpler,

$$
\begin{aligned}
& U_{t t}-U_{x x}-\frac{U_{x}}{x}=0, \\
& K_{t}=U_{t}+2 x U_{t} U_{x} \mp h, \\
& K_{x}=U_{x}+x\left(U_{t}^{2}+U_{x}^{2}\right)+h,
\end{aligned}
$$

which tell us that radiation fields can be generated from vaccuum spacetimes by adding a term $\mp H(t \mp x)$ to the conformal factor $K \underline{15}$.

This is the only case where a true axis might appear at $x=0$. But on applying the flatness condition in the vicinity of the axis 11,

$$
\lim _{x \rightarrow 0} \frac{\langle\operatorname{grad} \Delta, \operatorname{grad} \Delta\rangle}{4 \Delta}=\left.e^{2(U-K)}\right|_{x=0}=1, \quad \Delta=\left\langle\partial_{x}, \partial_{x}\right\rangle=x^{2} e^{2 U},
$$

assuming that the metric functions are regular at $x=0$, the quadrature equations for $K$, (21b 21c), would imply

$$
K_{t}(t, 0)=U_{t}(t, 0) \mp H_{t}(t), \quad K_{x}(t, 0)=U_{x}(t, 0) \mp H_{x}(t)
$$

naming $h=H^{\prime}$. Therefore $K(t, 0)=U(t, 0)+H(t)$ and condition (22) cannot be satisfied for all times unless $H$ is constant.

Furthermore, this means a problem in our parametrization, since the function $h(t \mp x)$ must be positive (negative) for positive (negative) values of $x$. Therefore, if $h\left(t_{0} \mp x_{0}\right)$ is positive for some values $x_{0}, t_{0}$, it should be positive for all values of $t$ and $h$ would be everywhere positive. It is obvious that there is no place at the same time for positive and negative values of $x$ and we have to resort, although there is no axis in strict sense, to the axial interpretation of the models and admit that $x$ ranges from zero to $\infty$ and 
it is a sort of radius. The coordinate $y$ would be interpreted as as an angle. In such case, $H_{t}=H^{\prime}=h$ must be positive everywhere and therefore the conformal factor $H$ is a growing function of its only variable.

- Similarly, if the gradient of $\rho$ is timelike, we may choose $\rho$ as a coordinate, $\rho=t$ without losing generality,

$$
\begin{aligned}
& U_{t t}-U_{x x}+\frac{U_{t}}{t}=0, \\
& K_{x}=U_{x}+2 t U_{t} U_{x} \mp h, \\
& K_{t}=U_{t}+t\left(U_{t}^{2}+U_{x}^{2}\right)+h,
\end{aligned}
$$

for which the results of the previous case are also valid.

\section{Geodesic completeness of the models}

The most usual definition of absence of singularities is causal geodesic completeness. This means that every timelike and lightlike geodesic, that is every physical trajectory of a test particle in the spacetime in the absence of external non-gravitatory forces, can be extended from $-\infty$ to $\infty$ in their affine parametrization, $x^{\mu}=x^{\mu}(\tau)$,

$$
\ddot{x}^{\mu}+\Gamma_{\nu \rho}^{\mu} \dot{x}^{\nu} \dot{x}^{\rho}=0, \quad g_{\mu \nu} \dot{x}^{\nu} \dot{x}^{\rho}=-\delta, \quad-\infty<\tau<\infty
$$

where $\delta$ takes the value zero for lightlike and one for timelike geodesics and the dot stands for derivation with respect to the affine parameter $\tau$. It is called affine parameter because it is unique but for affine transformations.

Causal geodesic completeness implies a simpler definition, regularity of the curvature scalars, but it is not true the other way round. Taub-NUT spacetime 16 is a nice counterexample for this 2]. Moreover, spacetimes where curvature scalars are regular may be incomplete because they can be extended to larger manifolds. Therefore in order to check geodesic completeness we are to analyse geodesic equations.

We have already seen that our models are classified in three sets according to the character of the gradient of $\rho$ :

- Lightlike gradient of $\rho$ : In this case the analysis of the geodesics becomes quite simple, since there are extra isometries, which allow us to reduce the order of the geodesic equations. We just need the three commuting generators $\left\{\partial_{v}, \partial_{y}, \partial_{z}\right\}$ to derive independent constants of geodesic motion,

$$
\begin{aligned}
V & =\left\langle\partial_{v}, \dot{x}\right\rangle=g_{v \mu} \dot{x}^{\mu}=-\dot{u}, \\
Y & =\left\langle\partial_{y}, \dot{x}\right\rangle=g_{y \mu} \dot{x}^{\mu}=F^{2} \dot{y}, \\
Z & =\left\langle\partial_{z}, \dot{x}\right\rangle=g_{z \mu} \dot{x}^{\mu}=G^{2} \dot{z},
\end{aligned}
$$


The first equation simply implies that $u$ is an acceptable affine parameter for the geodesics,

$$
u=u_{0}-V \tau \text {. }
$$

The second equation can be integrated for all values of $\tau$ or $u$,

$$
y=y_{0}-\frac{Y}{V} \int d u F^{-2}(u)
$$

provided that $F^{-2}$ is integrable for all values of $u$.

The third equation is also integrable if $G^{-2}$ is integrable for all values of $u$,

$$
z=z_{0}-\frac{Z}{V} \int d u G^{-2}(u)
$$

The remaining equation of geodesic motion needs not be derived from the Christoffel symbols but from the condition of affine parametrization,

$$
\delta=-2 V \dot{v}-Y^{2} F^{-2}-Z^{2} G^{-2},
$$

from which we do not get any additional restriction,

$$
\frac{d v}{d u}=\frac{1}{2 V^{2}}\left\{\delta+Y^{2} F^{-2}+Z^{2} G^{-2}\right\} .
$$

since $v$ is integrable provided that $y$ and $z$ are integrable.

These results may be summarized in the following theorem:

Theorem 1. An spacetime with a metric of the form (18) filled with a pure radiation field is causally geodesically complete if and only if the metric functions $g^{y y}$ and $g^{z z}$ are integrable for all values of the light coordinate $u$.

Examples of such spacetimes are easy to provide. For instance, if we resort to powers of the coordinate $u, F(u)=|u|^{\alpha / 2}, G(u)=|u|^{\beta / 2}$, the regularity requirement is fulfilled if $\alpha, \beta<1$. Since $\Phi$ has to be positive, an additional condition is imposed, $0<\alpha, \beta<2$. This example is intriguing since it states that half of the models are regular whereas the other half are singular.

Although the previous reasoning has been made for specific functions, it describes the behaviour of general functions $F$ and $G$ on becoming zero. In fact, since equation (19) imposes that the graphic of at least one of the functions must be convex, it is clear that the graphic of this function is to cut the axis at one point at least.

This poses a serious drawback on these spacetimes, since the previous analysis points out that the metric functions $F^{2}$ and $G^{2}$ are continuous, but not differentiable. We are dealing with topological manifolds instead of differentiable manifolds. However, metric invariants do not see this fact, since they are all null. 
- The analysis of models with non-lightlike gradient of $\rho$ is quite more complicated, since generically we do not have additional symmetries. Fortunately, we may resort to some theorems which have been explicitly devised for $G_{2}$ spacetimes 12 ,

Theorem: A diagonal Abelian orthogonally transitive spacetime with spacelike orbits endowed with a metric in the form (3) with $C^{2}$ metric functions $K, U, \rho$, where $\rho$ has a spacelike gradient, is future causally geodesically complete provided that along causal geodesics:

(1) For large values of $t$ and increasing $x$,

(a) $(K-U-\ln \rho)_{t}+(K-U-\ln \rho)_{x} \geq 0$, and either $(K-U-$ $\ln \rho)_{x} \geq 0$ or $\left|(K-U-\ln \rho)_{x}\right| \lesssim(K-U-\ln \rho)_{t}+(K-U-\ln \rho)_{x}$.

(b) $K_{t}+K_{x} \geq 0$, and either $K_{x} \geq 0$ or $\left|K_{x}\right| \lesssim K_{t}+K_{x}$.

(c) $(K+U)_{t}+(K+U)_{x} \geq 0$, and either $(K+U)_{x} \geq 0$ or $\mid(K+$ $U)_{x} \mid \lesssim(K+U)_{t}+(K+U)_{x}$.

(2) For large values of $t$, a constant $b$ exists such that

$$
\left.\begin{array}{c}
K(t, x)-U(t, x) \\
2 K(t, x) \\
x)+U(t, x)+\ln \rho(t, x)
\end{array}\right\} \geq-\ln |t|+b .
$$

For geodesics pointing to the past the theorem imposes the same conditions but for a sign in the time derivatives.

We just have to check the restrictions on the metric functions that such conditions require:

(1) Since $h$ is positive for radiation fields and positive values of $x$, we may reach some conclusions:

(a) $(K-U-\ln \rho)_{t}+(K-U-\ln \rho)_{x} \geq x\left(U_{t}+U_{x}\right)^{2}-1 / x$ is positive unless the derivatives of $U$ decrease too quickly. Such possibility is avoided if we impose

$$
x^{1-\varepsilon}\left|U_{x}+U_{t}\right| \not \supset 0,
$$

for large values of $t$ and $x$.

On the other hand $(K-U-\ln \rho)_{x} \geq x\left(U_{t}^{2}+U_{x}^{2}\right)-1 / x$ is also positive under the same conditions.

(b) $K_{t}+K_{x} \geq U_{t}+U_{x}+x\left(U_{t}+U_{x}\right)^{2}$ is again positive for large values of $t$ and $x$ if (34) is satisfied, as it happens with $K_{x} \geq U_{x}+x\left(U_{t}^{2}+U_{x}^{2}\right)$.

(c) The same restrictions are valid for $(K+U)_{t}+(K+U)_{x}$ and $\mid(K+$ $U)_{x} \mid$. Therefore (34) suffices for the satisfaction of the first part of the theorem.

For past-pointing geodesics, as it has already been told, condition 1 is the same for large negative values of $t$, but for the sign of the time 
derivatives. Therefore, the corresponding requirement for past-pointing geodesics is

$$
x^{1-\varepsilon}\left|U_{x}-U_{t}\right| \nrightarrow 00
$$

for small values of $t$ and large values of $x$.

(2) On $x=0$ we already know that $K(t, 0)-U(t, 0)=\mp H(t)$ and $H$ is a growing function. Therefore we may encounter a problem for outgoing radiation unless $H$ grows slower than a logarithm for large times,

$$
\mp H(t) \geq-\ln |t|+b .
$$

Out of $x=0$ the first condition of this set is satisfied,

$$
K(t, x)-U(t, x)=\mp H(t \mp x)+\int_{0}^{x} x^{\prime}\left\{U_{t}(t, x)^{2}+U_{x}(t, x)^{2}\right\} .
$$

since we are adding just a positive term to the previous case.

For the second condition on $x=0$, we obtain another constraint for large values of $t$,

$$
K(t, 0)=U(t, 0) \mp H(t) \geq-\frac{1}{2} \ln |t|+b,
$$

and out of $x=0$ it will be also satisfied.

Finally, for the third condition we may require,

$$
2 U(t, 0) \mp H(t) \geq-\ln |t|+b,
$$

always for large values of $t$.

These conditions on outgoing radiation may be summarized in the following way for large values of $t$,

$$
\begin{aligned}
H(t) & \leq \ln |t|+b \\
U(t, 0) & \geq b-\frac{1}{2} \ln |t|+H(t),
\end{aligned}
$$

since $H$ grows obviously faster than $H / 2$.

For ingoing radiation condition (36) is always fulfilled, since $H$ is a growing function. The only requirement which remains is

$$
U(t, 0) \geq b-\frac{1}{2} \ln |t|-\frac{H(t)}{2},
$$

for large values of $t$.

Similar restrictions are imposed for past-pointing geodesics for large negative values of $t$. The roles of outgoing and ingoing radiation are obviously exchanged. 
For outgoing radiation the only restriction in order to achieve causal geodesic completeness to the past is

$$
U(t, 0) \geq b-\frac{1}{2} \ln |t|+\frac{H(t)}{2},
$$

for small values of $t$, since $H / 2$ decreases to the past more slowly than $H$. On the other hand, the corresponding condition for ingoing radiation is

$$
\begin{aligned}
H(t) & \geq-\ln |t|+b \\
U(t, 0) & \geq b-\frac{1}{2} \ln |t|-H(t),
\end{aligned}
$$

for small values of $t$.

Summarizing the previous results, we may enclose them in two statements:

Theorem 2. A diagonal Abelian orthogonally transitive spacetime with spacelike orbits with a metric in the form (3) and a spatial gradient of $\rho$ and a pure outgoing radiation field as matter content is causally geodesically complete if:

(1) $x^{1-\varepsilon}\left|U_{x} \pm U_{t}\right| \not 0$ for large values of $|t|$ and $x$.

(2) $H(t) \leq \ln |t|+b$ and $U(t, 0) \geq b-\frac{1}{2} \ln |t|+H(t)$ for large values of $t$.

(3) $U(t, 0) \geq b-\frac{1}{2} \ln |t|+H(t) / 2$ for small values of $t$.

Theorem 3. A diagonal Abelian orthogonally transitive spacetime with spacelike orbits with a metric in the form (3) and a spatial gradient of $\rho$ and a pure ingoing radiation field as matter content is causally geodesically complete if:

(1) $x^{1-\varepsilon}\left|U_{x} \pm U_{t}\right| \nrightarrow 0$ for large values of $|t|$ and $x$.

(2) $U(t, 0) \geq b-\frac{1}{2} \ln |t|-H(t) / 2$ for large values of $t$.

(3) $H(t) \geq-\ln |t|+b$ and $U(t, 0) \geq b-\frac{1}{2} \ln |t|-H(t)$ for small values of $t$.

The meaning of such results seems clear. There must be an upper limit for $H$ in the future for outgoing radiation in order to prevent a huge amount of energy that could form a singularity. A symmetric reasoning can be applied to ingoing radiation.

$H$ can be any growing function, but $U$ is restricted by the 2-D wave equation (21a). Following 17, we may write the general solution of this equation in terms of initial data, $U(0, x)=f(x), U_{t}(0, x)=g(x)$,

$U(t, x)=\frac{1}{2 \pi} \int_{0}^{2 \pi} d \phi \int_{0}^{1} d \tau \frac{\tau}{\sqrt{1-\tau^{2}}}\left\{t g(v)+f(v)+t f^{\prime}(v) \frac{t \tau^{2}+x \tau \cos \phi}{v}\right\}$

where $v=\sqrt{x^{2}+t^{2} \tau^{2}+2 x t \tau \cos \phi}$. 
Since we are interested in the values of $U(t, 0)$, the expression we need is much simpler,

$$
U(t, 0)=\int_{0}^{1} d \tau \frac{\tau}{\sqrt{1-\tau^{2}}}\left\{t g(|t| \tau)+f(|t| \tau)+|t| \tau f^{\prime}(|t| \tau)\right\},
$$

which allows easy implementation of the regularity conditions.

For instance, we can construct non-singular radiation models just prescribing a function $U$ which grows for large values of $|t|$ and $x$ and a function $H$ that does not grow or decrease too fast, that is, bounded by a logarithm.

A simple example of a way to comply with the regularity requirements for $U$ is shown in 9 for polynomial initial data: if $f(x)=\sum a_{i} x^{i}$ and $g(x)=\sum b_{j} x^{j}$ are polynomials in $x$ of degrees $m, n$, respectively, $U$ fulfills the regularity conditions if $a_{m}>0$ and $m>n+1$ or if $m=n+1$ and $(m+1 / 2) a_{m}>\left|b_{n}\right|$.

- We shall not consider causal geodesic completeness in the case of a timelike gradient of $\rho$ since the logarithm term of the time coordinate in the regularity theorems would mean a singularity at $t=0$.

\section{Discussion}

We have discussed in this paper the possibility of constructing regular inhomogenous spacetimes with a pure radiation field as matter content. It has been shown that such cosmological models are very easy to construct in terms of a nearly arbitrary growing function $H$, which is bounded by a logarithmic function, and a solution of a wave equation, $U$, with rather few limitations. The conclusions are close to the results for stiff perfect fluids: pure radiation inhomogeneous cosmological models are an infinite family and are not negligible in the set of much models.

Pure radiation fields satisfy all energy conditions and these models are causally

stable since the function $t$ has a positive gradient in all the spacetime 2 . Therefore these models fulfill weaker causality conditions suchs as the chronology conditions. As it happened in most regular cosmological models, the way to elude singularity theorems is the lack of trapped sets.

The existence of such an amount of non-singular cosmological models fulfilling all energy and chronology conditions suggests that they are more abundant than it was thought before. If they had not been found previously it was due merely to the techniques used to generate them, not to their scarcity among inhomogeneous cosmological models. It remains however an open question whether such non-singular behaviour is just a peculiarity of $G_{2}$ inhomogeneous models and if these results can be extended to smaller groups of isometries or to other matter contents.

As a curiosity, it is interesting to notice that perfectly regular spacetimes can be turned singular by introducing radiation. For instance, the metric,

$$
d s^{2}=e^{r-t}\left\{-d t^{2}+d r^{2}\right\}+r^{2} d \phi^{2}+d z^{2},
$$


can be generated from Minkowski spacetime by adding a conformal factor. It is easy to check that axial geodesics are not complete,

$$
\begin{gathered}
r=0, \quad \dot{\phi}=0, \quad \delta=e^{-t} \dot{t}^{2}-Z^{2} \\
\dot{t}=\sqrt{\delta+Z^{2}} e^{t} \Rightarrow t(\tau)=-\ln \left(\sqrt{\delta+Z^{2}}(k-\tau)\right) .
\end{gathered}
$$

\section{Acknowledgments}

The present work has been supported by Dirección General de Enseñanza Superior Project PB98-0772. The authors wish to thank F.J. Chinea and F. Navarro-Lérida for valuable discussions.

\section{References}

1. F. Hoyle, J.V. Narlikar, Proc. Roy. Soc. Lon. A 282, 191, (1964)

2. S. W. Hawking, G. F. R. Ellis, The Large Scale Structure of Space-time, Cambridge University Press, Cambridge, (1973)

3. J. Beem, P. Ehrlich, K. Easley, Global Lorentzian Geometry, Dekker, New York, (1996)

4. R. Penrose, Phys. Rev. Lett. 14, 57 (1965)

5. S.W. Hawking, R. Penrose, Proc. Roy. Soc. Lon. A 314, 529, (1970)

6. J. M. M. Senovilla, Phys. Rev. Lett. 64, 2219, (1990)

7. J.M.M. Senovilla, Gen. Rel. Grav. 30, 701 (1998)

8. J.M.M. Senovilla, "Singularity-Free Spacetimes". In: El Escorial Summer School on Gravitation and General Relativity 1992: Rotating Objects and Other Topics (eds.: F. J. Chinea and L.M. González-Romero), Springer-Verlag, Berlin-New York (1993)

9. L. Fernández-Jambrina, L.M. González-Romero, Phys. Rev. D 66, 024027 (2002)

10. E. Ruiz, J. M. M. Senovilla, Phys. Rev D45, 1995, (1992)

11. H. Stephani, D. Kramer, M. MacCallum, C. Hoenselaers, E. Herlt, Exact Solution's of Einstein's Field Equations, 2nd edition, Cambridge University Press, Cambridge (2003)

12. L. Fernández-Jambrina, L.M. González-Romero, Class. Quantum Grav. 16, 953, (1925)

13. H.W. Brinkmann, Math. Ann., 18, 119 (1925)

14. O.R. Baldwin, G.B. Jeffery, Proc. Roy. Soc. Lond., A 111, 95 (1926)

15. J. Krishna Rao, Proc. Nat. Inst. Sci. India A 30, 439, (1964)

16. E.T. Newman, L.A. Tamburino, T. Unti, Journ. Math. Phys. 4, 915 (1963)

17. F. John, Partial Differential Equations, 4 edition, Springer-Verlag, Berlin-New York (1982) 